\title{
DESCRIPTION OF FACIALLY SYMMETRIC SPACES WITH UNITARY TRIPOTENTS
}

\author{
KARIMBERGEN KUDAYBERGENOV AND JUMABEK SEYPULLAEV
}

\begin{abstract}
We give a description of finite-dimensional real neutral strongly facially symmetric spaces with the property JP. We also prove that if $Z$ is a real neutral strongly facially symmetric with an unitary tripotents, then the space $Z$ is isometrically isomorphic to the space $L_{1}(\Omega, \Sigma, \mu)$, where $(\Omega, \Sigma, \mu)$ is a measure space having the direct sum property.

Keywords: strongly facially symmetric space, norm exposed face, geometric tripotent, geometric Peirce projections
\end{abstract}

\section{INTRODUCTION}

A geometric characterization of state spaces of operator algebras is important problem of the theory of operator algebras In the middle of 1980s, Friedman and Russo wrote the paper [1, 2] related to this problem, in which they introduced facially symmetric spaces, largely for the purpose of obtaining a geometric characterization of the predual spaces of $\mathrm{JBW}^{*}$-triples admitting an algebraic structure. Many of the properties required in these characterizations are natural assumptions for state spaces of physical systems. Such spaces are regarded as a geometric model for states of quantum mechanics. In [3] was proved that the preduals of complex von Neumann algebras and, more generally, complex $\mathrm{JBW}^{*}$-triples are neutral strongly facially symmetric spaces.

The project of classifying facially symmetric spaces was initiated in [4, where a geometric characterization of complex Hilbert spaces and complex spin factors was given. Moreover, there were described the $\mathrm{JBW}^{*}$-triples of ranks 1 and 2 and Cartan factors of types 1 and 4. Afterwards, Friedman and Russo obtained a description of atomic facially symmetric spaces [5]. Namely, they showed that a neutral strongly facially symmetric space is linearly isometric to the predual of one of the Cartan factors of types 1-6 provided that it satisfies four natural physical axioms, which hold for the predual spaces of $\mathrm{JBW}^{*}$-triples.

In paper [10], Neal and Russo found geometric conditions under which a facially symmetric space is isometric to the predual of a $\mathrm{JBW}^{*}$-triple. In particular, they proved that any neutral strongly facially symmetric space decomposes into a direct sum of atomic and nonatomic strongly facially symmetric spaces. In [6], a complete description of strongly facially symmetric spaces that are isometrically isomorphic to a predual space atom commutative von Neumann algebra.

In [12, Theorem 3.2] was established that if $Z$ is a real neutral strongly facially symmetric space with unitary tripotent such that any maximal tripotent is unitary, then $Z$ is isometrically isomorphic to $L_{1}$-space. In the present paper shows that the condition "every maximal tryptotent is unitary "in the above theorem is superfluous. We also we give a description of finite-dimensional real neutral SFS-spaces with the property JP. 


\section{FACially Symmetric SPACES.}

In this section we shall recall some basic facts and notation about facially symmetric spaces (see for details [1, 2]).

Let $Z$ be a real or complex normed space. Elements $f, g \in Z$ are orthogonal, notation $f \diamond g$, if $\|f+g\|=\|f-g\|=\|f\|+\|g\|$. Subset $S, T \subset Z$ are said to be orthogonal, notation $S \diamond T$, if $f \diamond g$ for all $(f, g) \in S \times T$. A norm exposed face of the unit ball $Z_{1}=\{f \in Z:\|f\| \leq 1\}$ of $Z$ is a non-empty set (necessarilly $\neq Z_{1}$ ) of the form $F_{u}=\left\{f \in Z_{1}: u(f)=1\right\}$, where $u \in Z^{*},\|u\|=1$. Recall that a face $F$ of a convex set $K$ is a non-empty convex subset of $K$ such that if $f \in F$ and $g, h \in K$ satisfy $f=\lambda g+(1-\lambda) h$ for some $\lambda \in(0,1)$, then $g, h \in F$. In particular, an extreme point of $K$ is face of $K$. An element $u \in Z^{*}$ is called a projective unit if $\|u\|=1$ and $\left\langle u, F_{u}^{\diamond}\right\rangle=0$. Here, for any subset $S, S^{\diamond}$ denotes the set of all elements orthogonal to each elements of $S$. A norm exposed face $F_{u}$ in $Z_{1}$ is said to be a symmetric face if there is a linear isometric symmetry $S_{u}$ of $Z$ onto $Z$, with $S_{u}^{2}=I$ such that fixed point set of $S_{u}$ is $\left(\overline{\mathrm{sp}} F_{u}\right) \oplus F_{u}^{\diamond}$.

A real or complex normed space $Z$ is said weakly facially symmetric (WFS) if every norm exposed face in $Z_{1}$ is symmetric.

For each symmetric face $F_{u}$ we defined contractive projections $P_{k}\left(F_{u}\right), k=0,1,2$ on $Z$ as follows. First, $P_{1}\left(F_{u}\right)=\left(I-S_{u}\right) / 2$ is the projection on the -1 eigenspace of $S_{u}$. Next we define $P_{2}\left(F_{u}\right)$ and $P_{0}\left(F_{u}\right)$ as the projections of $Z$ onto $\overline{\mathrm{sp}} F_{u}$ and $F_{u}^{\diamond}$, respectively. A geometric tripotent is a projective unit $u \in Z^{*}$ with the property that $F_{u}$ is a symmetric face and $S_{u}^{*} u=u$ for a symmetry $S_{u}$ corresponding to $F_{u}$. The projections $P_{k}\left(F_{u}\right)$ are called geometric Peirce projections.

By $\mathcal{G U}$ and $\mathcal{S F}$ denote the collections of geometric tripotents and symmetric faces respectively, and the map $\mathcal{G U} \ni u \mapsto F_{u} \in \mathcal{S F}$ is a bijection [2, Proposition 1.6]. For each geometric tripotent $u$ in the dual of a WFS space $Z$, we shall denote the geometric Peirce projections by $P_{k}(u)=P_{k}\left(F_{u}\right), k=0,1,2$. We set $U=Z^{*}, Z_{k}(u)=P_{k}(u) Z, U_{k}(u)=$ $P_{k}(u)^{*} Z^{*}$. The Peirce decomposition

$$
Z=Z_{2}(u)+Z_{1}(u)+Z_{0}(u), U=U_{2}(u)+U_{1}(u)+U_{0}(u) .
$$

Tripotents $u$ and $v$ are said to be orthogonal if $u \in U_{0}(v)$ (which implies $v \in U_{0}(u)$ ) or, equivalently, $u \pm v \in \mathcal{G T}$ (see [1, Lemma 2.5]). More generally, elements $a$ and $b$ of $U$ are said to be orthogonal if one of them belongs to $U_{2}(u)$ and the other belongs to $U_{0}(u)$ for some geometric tripotent $u$.

A contractive projection $Q$ on $Z$ is said to be neutral if $\|Q f\|=\|f\|$ implies $Q f=f$ for each $f \in Z$. A normed space $Z$ is said to be neutral if, for every symmetric face $F_{u}$, the projection $P_{2}(u)$ is neutral.

A WFS space $Z$ is strongly facially symmetric (SFS) if for every norm exposed face $F_{u}$ in $Z_{1}$ and every $y \in Z^{*}$ with $\|y\|=1$ and $F_{u} \subset F_{y}$, we have $S_{u}^{*} y=y$, where $S_{u}$ denotes a symmetry associated with $F_{u}$.

The principal examples of neutral complex strongly facially symmetric spaces are preduals of complex $\mathrm{JBW}^{*}$-triples, in particular, the preduals of complex von Neumann algebras (see [3]). In a neutral strongly facially symmetric space $Z$, every non-zero element has a polar decomposition [2, Theorem 4.3]: for non-zero $f \in Z$ there exists a unique geometric tripotent $v=v_{f}$ with $\langle v, f\rangle=\|f\|$ and $\left\langle v, f^{\diamond}\right\rangle=0$. If $f, g \in Z$, then $f \diamond g$ if and only if $v_{f} \diamond v_{g}$, as follows from [1, Corollary 1.3(b) and Lemma 2.1]. A partial ordering can be defined on the set of geometric tripotents as follows: if $u, v \in \mathcal{G} \mathcal{T}$, then 
$u \leq v$ if $F_{u} \subset F_{v}$, or equivalently, by [2, Lemma 4.2], $P_{2}(u)^{*} v=u$, or $v-u$ is either zero or a geometric tripotent orthogonal to $u$. As is known [2, Proposition 4.5], the set $L_{e}=\{v \in \mathcal{G} \mathcal{T}: v \leq e\} \cup\{0\}$ is a complete orthomodular lattice with orthocomplement $u^{\perp}=e-u$, with respect to the order " $\leq$ ". The convex hull of the points $x_{1}, x_{2}, \ldots, x_{n+1}$ in general position is called an $n$-dimensional simplex. In a finite-dimensional neutral SFS-space Z, every norm exposed face is a simplex (see [9, Proposition 1]).

\section{DeSCRIPTION OF Finite-DIMENSIONAL REAL STRONGLY FACIALLY SYMMETRIC SPACES}

One of the important concepts in SFS-spaces is the notion of rank of space, which was introduced in [5]. In [7] was given a description of a unit ball of an $n$-dimensional real SFS-space of rank $n$. In [11] was obtained a description of the unit ball of a reflexive SFS-space of rank 1. In addition, in the [9] was given a description of a unit ball of an $n$-dimensional real neutral SFS-space of rank $n-1$. The problem of characterizing SFSspaces with respect to rank remains is still open. In this section we give a description of finite-dimensional real neutral SFS-spaces with the property JP.

A facially symmetric space $Z$ is of $\operatorname{rank} n(n=1,2, \ldots)$, notation $\operatorname{rank} Z=n$, if every orthogonal family of geometric tripotents has cardinality at most $n$, and if there is at least one orthogonal family of geometric tripotents containing exactly $n$ elements (see [5]).

A WFS space $Z$ satisfies JP (joint Peirce decomposition) if for any pair $u, v$ of orthogonal geometric tripotents, we have $S_{u} S_{v}=S_{u+v}$ where for any geometric tripotent $w, S_{w}$ is the symmetry associated with the symmetric face $F_{w}$ (see [5]).

The space $\mathbb{R}^{n}$ with the norm

$$
\|f\|=\sum_{i=1}^{k} \sqrt{\sum_{j=n_{i}}^{n_{i+1}} f_{j}^{2}},
$$

where $1=n_{1}<n_{2}<\ldots<n_{k+1}=n$, is a SFS-space of rank $k$ and satisfies JP.

The following theorem gives a description of finite-dimensional real neutral SFS-spaces with the property JP.

Theorem 3.1. Let $Z$ be a finite-dimensional real neutral strongly facially symmetric space with property JP. Then

$$
Z \cong \bigoplus_{i=1}^{k} H_{i}
$$

where $H_{i}$ is a Hilbert space, $i \in \overline{1, k}$ and $k=\operatorname{rank} Z$.

Recall that a face $F$ of a convex set $K$ is called a split face if there exists a face $G$, called complementary to $F$, such that $F \cap G=\emptyset$ and $K$ is the direct convex sum $F \oplus_{c} G$, i.e. any element $f \in K$ can be uniquely represented in the form $f=t g+(1-t) h$, where $t \in[0 ; 1] f \in F, h \in G$.

In this section we assume that $Z$ is a finite-dimensional real neutral SFS-space with the property JP.

For the proof we need several lemmas.

Lemma 3.2. If $u$ and $v$ are orthogonal geometric tripotents, then

(i) $F_{u+v}=F_{u} \oplus_{c} F_{v}$; 
(ii) $P_{2}(u+v)=P_{2}(u)+P_{2}(v)$;

(iii) $P_{1}(u) P_{1}(v)=0$.

Proof. (i) Let $u \diamond v, u, v \in \mathcal{G} \mathcal{T}$. Then, by [1, lemma 2.5] we get that $F_{u} \diamond F_{v}$. Therefore, taking into account that in a real finite-dimensional SFS-space $Z$ every norm exposed face is a simplex (see [9, proposition 1]), then $F_{u} \oplus_{c} F_{v}$ is a symmetric face. Moreover, by [1, lemma 2.1] it follows that $F_{u}, F_{v} \subset F_{u+v}$ and therefore $F_{u} \oplus_{c} F_{v} \subset F_{u+v}$. Then by [1, lemma 2.7] it follows that $F_{u} \oplus_{c} F_{v}=F_{u+v}$, or $\left(F_{u} \oplus_{c} F_{v}\right)^{\diamond} \cap F_{u+v} \neq \emptyset$.

Suppose that $0 \neq f \in\left(F_{u} \oplus_{c} F_{v}\right)^{\diamond} \cap F_{u+v}$. Then $f \in F_{u}^{\diamond}$ and $f \in F_{v}^{\diamond}$. Therefore $f \in F_{u}^{\diamond} \cap F_{v}^{\diamond}$. Then, by [1, lemma 1.8] it follows that $f \in F_{u}^{\diamond} \cap F_{v}^{\diamond}=F_{u+v}^{\diamond}$. It contradicts the fact that $f \in F_{u+v}$. Hence, $F_{u+v}=F_{u} \oplus_{c} F_{v}$.

(ii) Since $Z$ is a finite-dimensional SFS-space, then from (i) follows (ii).

(iii) Let $u \diamond v, u, v \in \mathcal{G} \mathcal{T}$. Since $Z$ has the property of JP, then by [5, Remark 4.2] it follows that

$$
P_{2}(u+v)=P_{2}(u)+P_{2}(v)+P_{1}(u) P_{1}(v) .
$$

On the other hand, from (ii) we have

$$
P_{2}(u+v)=P_{2}(u)+P_{2}(v) .
$$

This means that $P_{1}(u) P_{1}(v)=0$. The proof is complete.

Lemma 3.3. For every $u \in \mathcal{G} \mathcal{T}$, we have

$$
Z_{0}(u) \diamond\left(Z_{1}(u)+Z_{2}(u)\right) .
$$

Proof. Let $f \in Z_{1}(u)$ and $g \in Z_{0}(u)$, then by [4, Corollary 2.2] it follows that $v_{f} \in U_{1}(u)$ and $v_{g} \in U_{0}(u)$. Then by [2, Corollary 3.4] it follows that $P_{2}\left(v_{g}\right)^{*} P_{1}(u)^{*}=0$, and by lemma 1 we have $P_{1}\left(v_{g}\right)^{*} P_{1}(u)^{*}=0$. Using these relations, we obtain

$$
\begin{array}{r}
v_{f}=P_{1}(u)^{*} v_{f}=\left[P_{2}\left(v_{g}\right)^{*}+P_{1}\left(v_{g}\right)^{*}+P_{0}\left(v_{g}\right)^{*}\right] P_{1}(u)^{*} v_{f}= \\
=P_{2}\left(v_{g}\right)^{*} P_{1}(u)^{*} v_{f}+P_{1}\left(v_{g}\right)^{*} P_{1}(u)^{*} v_{f}+P_{0}\left(v_{g}\right)^{*} P_{1}(u)^{*} v_{f}= \\
=P_{0}\left(v_{g}\right)^{*} P_{1}(u)^{*} v_{f}=P_{0}\left(v_{g}\right)^{*} v_{f} .
\end{array}
$$

Hence $v_{f} \in U_{0}\left(v_{g}\right)$, and therefore, by the orthogonality of geometric tripotents, we have $v_{f} \diamond v_{g}$. Hence, $f \diamond g$. This means that $Z_{1}(u) \diamond Z_{0}(u)$. In addition, by [2, proposition 1.5] it follows that $Z_{2}(u) \diamond Z_{0}(u)$. Therefore, by [2, proposition 1.1] $Z_{0}(u) \diamond\left(Z_{1}(u)+Z_{2}(u)\right)$. The proof is complete.

A geometric tripotent $u$ is called maximal if $v \in \mathcal{G} \mathcal{T}$ from $u \leq v$ implies $v=u$ or is equivalent to $P_{0}(u)=0$.

Lemma 3.4. Let $u, v \in \mathcal{G T}$ and $u \diamond v$. If $u+v$ is maximal, then

(i) $P_{0}(u) P_{0}(v)=0$;

(ii) $Z_{0}(u)=Z_{2}(v)+Z_{1}(v)$;

(iii) $Z=Z_{0}(u) \oplus Z_{0}(v)$.

Proof. (i) If $u$ and $v$ are mutually orthogonal geometric tripotents, it follows from [2, lemma 1.8] that $P_{0}(u) P_{0}(v)=P_{0}(u+v)$. Therefore, from the maximality $u+v$ we have $P_{0}(u) P_{0}(v)=P_{0}(u+v)=0$. 
(ii) Let $f \in Z_{0}(u)$. Then by [2, Corollary 3.4],

$$
\begin{aligned}
f & =\left[P_{2}(v)+P_{1}(v)+P_{0}(v)\right] P_{0}(u) f= \\
& =P_{2}(v) P_{0}(u) f+P_{1}(v) P_{0}(u) f+P_{0}(v) P_{0}(u) f= \\
& =P_{2}(v) f+P_{1}(v) f .
\end{aligned}
$$

Thus $f \in\left(Z_{2}(v)+Z_{1}(v)\right)$.

Let $f \in Z_{2}(v)+Z_{1}(v)$. Since $P_{1}(u) P_{1}(v)=0$ and $P_{0}(u) P_{0}(v)=0$, then by [2, Corollary 3.4 (a)] we have that

$$
\begin{aligned}
f & =\left[P_{2}(u)+P_{0}(u)+P_{1}(u)\right]\left(P_{2}(v) f+P_{1}(v) f\right)= \\
& =P_{2}(u) P_{2}(v) f+P_{0}(u) P_{2}(v) f+P_{1}(u) P_{2}(v) f+ \\
& +P_{2}(u) P_{1}(v) f+P_{0}(u) P_{1}(v) f+P_{1}(u) P_{1}(v) f= \\
& =P_{0}(u) P_{2}(v) f+P_{0}(u) P_{1}(v) f=P_{0}(u)\left[P_{2}(v)+P_{1}(v)\right] f=P_{0}(u) f .
\end{aligned}
$$

Therefore, $f \in Z_{0}(u)$. Hence, $Z_{0}(u)=Z_{2}(v)+Z_{1}(v)$.

(iii) Since $\left(Z_{2}(v)+Z_{1}(v)\right) \diamond Z_{0}(v)$ and $Z_{0}(u)=Z_{2}(v)+Z_{1}(v)$, then the Pierce expansion can be rewritten as $Z=Z_{0}(u) \oplus Z_{0}(v)$. The proof is complete.

Lemma 3.5. Let $Z$ be a neutral strongly facially symmetric space with the property JP. Then for every $u \in \mathcal{G} \mathcal{T}$, the subspace $Z_{0}(u)$ has the property $J P$.

Proof. Let $u \in \mathcal{G} \mathcal{T}$, then by [2, Proposition 4.1] the subspace $Z_{0}(u)$ is a neutral SFSspace. We denote by $\mathcal{G} \mathcal{T}_{Z_{0}(u)}$ and $\mathcal{S} \mathcal{F}_{Z_{0}(u)}$ the set of all geometric tripotents of $U_{0}(u)$ and symmetric faces in $Z_{0}(u)$.

Let $u_{1}, u_{2} \in \mathcal{G T}_{Z_{0}(u)}$ and $u_{1} \diamond u_{2}$. By the Hahn-Banach theorem, we extend the functionals $u_{1}, u_{2}$ to $Z$, which we denote by $x_{1}, x_{2}$, preserving the norm, respectively. Then

$$
\left\|x_{i}\right\|=1, \quad x_{i}(f)=u_{i}(f), \quad \forall f \in Z_{0}(u),\left.\quad x_{i}\right|_{Z_{2}(u)+Z_{1}(u)}=0, \quad i=1,2 .
$$

Thus, by [2, Theorem 2.3], there exist geometric tripotents $v_{i} \in \mathcal{G} \mathcal{T} \cap U_{0}(u)$ such that $F_{x_{i}}=F_{v_{i}}$, where $i=1,2$. Then by (1), we have

$$
F_{v_{i}}=F_{u_{i}}, \quad v_{i}(f)=u_{i}(f) \forall f \in Z_{0}(u),\left.\quad v_{i}\right|_{Z_{2}(u)+Z_{1}(u)}=0, \quad i=1,2 .
$$

Further [2, Theorem 3.6 and Proposition 4.1], imply that

$$
P_{k}\left(u_{i}\right):=\left.P_{k}\left(v_{i}\right)\right|_{Z_{0}(u)}, \quad S_{u_{i}}:=\left.S_{v_{i}}\right|_{Z_{0}(u)},
$$

where $k=0,1,2, i=1,2$.

Now we show that $v_{1} \diamond v_{2}$ and $S_{u_{1}+u_{2}}=\left.S_{v_{1}+v_{2}}\right|_{Z_{0}(u)}$. Since $u_{1} \diamond u_{2}, v_{i} \in \mathcal{G} \mathcal{T} \cap U_{0}(u)$, then by virtue of [2, Theorem 3.6 and Corollary $3.4(\mathrm{a})]$ we have $P_{2}\left(u_{2}\right) P_{2}\left(u_{1}\right)=P_{2}\left(u_{1}\right) P_{2}\left(u_{2}\right)=0$ and $P_{0}(u) P_{2}\left(v_{i}\right)=P_{2}\left(v_{i}\right) P_{0}(u)=P_{2}\left(v_{i}\right)$, where $i=1,2$. Therefore, by (2) and (3), for any $f \in Z$ we have

$$
\begin{aligned}
\left\langle P_{2}\left(v_{1}\right)^{*} v_{2}, f\right\rangle & =\left\langle v_{2}, P_{2}\left(v_{1}\right) f\right\rangle=\left\langle u_{2}, P_{2}\left(v_{1}\right) f\right\rangle= \\
& =\left\langle P_{2}\left(u_{2}\right)^{*} u_{2}, P_{2}\left(v_{1}\right) f\right\rangle=\left\langle u_{2}, P_{2}\left(u_{2}\right) P_{2}\left(v_{1}\right) P_{0}(u) f\right\rangle= \\
& =\left\langle u_{2}, P_{2}\left(u_{2}\right) P_{2}\left(u_{1}\right) P_{0}(u) f\right\rangle=\left\langle u_{2}, 0\right\rangle=0 .
\end{aligned}
$$

Then $P_{2}\left(v_{1}\right)^{*} v_{2}=0$. Analogously, we get that $P_{1}\left(v_{1}\right)^{*} v_{2}=0$. Hence, $P_{0}\left(v_{1}\right)^{*} v_{2}=v_{2}$. Therefore, $v_{1} \diamond v_{2}$. Then by (2) the geometric tripotent $v_{1}+v_{2}$ is an extension of $u_{1}+u_{2}$ and $F_{v_{1}+v_{2}}=F_{u_{1}+u_{2}}$. Thus, by virtue of [2, Theorem 3.6 and Proposition 4.1], we have

$$
S_{u_{1}+u_{2}}:=\left.S_{v_{1}+v_{2}}\right|_{Z_{0}(u)} \text {. }
$$


Since $Z$ has the property of JP, it follows from (3) and (4) that we have

$$
S_{u_{1}+u_{2}}=\left.S_{v_{1}+v_{2}}\right|_{Z_{0}(u)}=\left.\left.S_{v_{1}}\right|_{Z_{0}(u)} S_{v_{2}}\right|_{Z_{0}(u)}=S_{u_{1}} S_{u_{2}} .
$$

Hence, $Z_{0}(u)$ has the property JP. The proof is complete.

Proof of Theorem 3.1. In the case when $\operatorname{dim} Z=2$ and $\operatorname{dim} Z=3$, the statement of theorem follows from [9, Proposition 1 and Theorem 2].

Suppose that the theorem is true when $\operatorname{dim} Z<n$. Let $\operatorname{dim} Z=n$ and $\operatorname{rank} Z=$ $k$. Then by the definition of the rank of the space there exists a maximal family of mutually orthogonal geometric tripotents $u_{1}, u_{2}, \ldots, u_{k}$, and by [1, lemma 2.5] follows that $u_{1}+u_{2}+\ldots+u_{k}$ is a maximal geometric tripotent. Then by lemma 3 it follows that

$$
Z=Z_{0}(u) \oplus Z_{0}(v), \quad Z_{0}(u) \diamond Z_{0}(v),
$$

where $u=u_{1}, v=u_{2}+\ldots+u_{k}$.

By [2, Proposition 4.1] and Lemma 4 the subspaces $Z_{0}(u)$ and $Z_{0}(v)$ are neutral SFSspaces satisfying JP with dimensions less that $n$. By the assumption of induction these are direct sums of Hilbert spaces. By (5) it follows that $Z$ is also a direct sum of Hilbert spaces. The proof is complete.

Thus, unlike the case when $\operatorname{rank} Z=1, n-1, n$ (in each of these of cases, up to isometrically isomorphism, there is one space), in the case $1<\operatorname{rank} Z<n-1$ the number of non-isomorphic spaces is at least $\left[\frac{n}{k}\right]$, where $k=\operatorname{rank} Z,[t]$ is the integer part of the number $t$. The number of non-isomorphic spaces can be calculated from the recurrence relation

$$
p(n)=\sum_{m=1}^{\infty}(-1)^{m+1}\left(p\left(n-\frac{m(3 m-1)}{2}\right)+p\left(n-\frac{m(3 m+1)}{2}\right)\right) .
$$

\section{Description of FACIALly SYMmetric SPACES With UNITARY TRIPOTENTS}

A geometric tripotent $e \in \mathcal{G} \mathcal{T}$ is said to be unitary if the convex hull of the set $F_{e} \cup F_{-e}$ coincides with the unit ball $Z_{1}$, i.e.

$$
Z_{1}=\operatorname{co}\left(F_{e} \cup F_{-e}\right) \text {. }
$$

Also note that property (6) is much stronger than the Jordan decomposition property of a norm exposed face (see [10, Lemmata 2.3]). Recall that a norm exposed face $F_{u}$ has the Jordan decomposition property if its real span coincides with the geometric Peirce 2-space of the geometric tripotent $u$.

The space $\mathbb{R}^{n}$ with the norm $\|x\|=\sum_{i=1}^{n}\left|x_{i}\right|, x=\left(x_{i}\right) \in \mathbb{R}^{n}$ is a SFS space. If $e \in \mathbb{R}^{n} \cong$ $\left(\mathbb{R}^{n}\right)^{*}$ is a maximal geometric tripotent then $e=\left(\varepsilon_{1}, \ldots, \varepsilon_{n}\right), \varepsilon_{i} \in\{-1,1\}, i=\overline{1, n}$, and in this case the norm exposed face

$$
F_{e}=\left\{x \in \mathbb{R}^{n}: \sum_{i=1}^{n} \varepsilon_{i} x_{i}=1, \varepsilon_{i} x_{i} \geq 0, i=\overline{1, n}\right\}
$$

satisfies (6).

More generally, consider a measure space $(\Omega, \Sigma, \mu)$ with measure $\mu$ having the direct sum property, i.e. there is a family $\{\Omega\}_{i \in J} \subset \Sigma, 0<\mu\left(\Omega_{i}\right)<\infty, i \in J$, such that for any $A \in \Sigma$ with $\mu\left(\Omega_{i}\right)<\infty$, there exist a countable subset $J_{0} \subset J$ and a set $B$ of zero measure such that $A=\bigcup_{i \in J_{0}}\left(A \cap \Omega_{i}\right) \cup B$. 
Let $L_{1}(\Omega, \Sigma, \mu)$ be the space of all real integrable functions on $(\Omega, \Sigma, \mu)$. The space $L_{1}(\Omega, \Sigma, \mu)$ with the norm $\|f\|=\int_{\Omega}|f(t)| d \mu(t), f \in L_{1}(\Omega, \Sigma, \mu)$, is a SFS space. If $e \in L^{\infty}(\Omega, \Sigma, \mu) \cong L_{1}(\Omega, \Sigma, \mu)^{*}$, is a maximal geometric tripotent then $e=\tilde{\chi}_{A}-\tilde{\chi}_{\Omega \backslash A}$ for some $A \in \Sigma$, where $\widetilde{\chi}_{A}$ is the class containing the indicator function of the set $A \in \Sigma$. Then the norm exposed face

$$
F_{e}=\left\{f \in L_{1}(\Omega, \Sigma, \mu):\|f\|=1, \int_{\Omega} e(t) f(t) d \mu(t)=1\right\}
$$

satisfies (6).

In [12], Theorem 3.2 it was established that if $Z$ is a real neutral strongly facially symmetric space with unitary tripotent such that any maximal tripotent is unitary, then $Z$ is isometrically isomorphic to $L_{1}$-space.

The following result shows that the condition "every maximal triptotent is unitary" in the above theorem is superfluous.

Lemma 4.1. Let $Z$ is a real neutral SFS-space with unitary tripotent. Then every maximal tripotent is unitary.

Proof. Let $e$ be a unitary geometric tripotent and $u$ is maximal geometric tripotent. Then by [12, Lemma 3.4], there exist mutually orthogonal geometric tripotents $u_{1}, u_{2} \leq e$ such that $u=u_{1}-u_{2}$. Since $u$ is a maximal tripotent, then by [12, Lemma 3.5] we get that $u_{1}+u_{2}$ is a maximal geometric tripotent.

On the other side, if $u_{1}, u_{2} \leq e$ are mutually orthogonal geometric tripotents, then by [1. Lemma 2.1] we obtain that $F_{u_{1}}, F_{u_{2}} \subset F_{u_{1}+u_{2}}$, consequently $u_{1}, u_{2} \leq u_{1}+u_{2}$. Since, $L_{e}=\{v \in \mathcal{G U}: v \leq e\} \cup\{0\}$ is a complete orthomodular lattice (see [2, Proposition 4.5]), then $u_{1}+u_{2} \leq e$. Therefore, by the maximality of $u_{1}+u_{2}$ it follows that $e=u_{1}+u_{2}$. Then $F_{u_{1}}=F_{u} \cap F_{e}$ and $F_{-u_{2}}=F_{u} \cap F_{-e}$.

Now, we show that

$$
F_{u}=F_{u_{1}} \oplus_{c} F_{-u_{2}}
$$

For this it suffices to show that $F_{u} \subseteq F_{u_{1}} \oplus_{c} F_{-u_{2}}$.

Let $f \in F_{u}$. Then, from equality (6) we obtain $f=t g+(1-t) h$ where $g \in F_{e}, h \in F_{-e}$, $0 \leq t \leq 1$.

If $t=1$, then $f=g$, and therefore $f \in F_{u} \cap F_{e}=F_{u_{1}}$.

If $t=0$, then $f=h$, and therefore $f \in F_{u} \cap F_{-e}=F_{-u_{2}}$.

Now let $0<t<1$. Since $F_{u}$ is a face, then $g, h \in F_{u}$. Therefore $g \in F_{u} \cap F_{e}=F_{u_{1}}$, $h \in F_{u} \cap F_{-e}=F_{-u_{2}}$. Thus, from $F_{u_{1}} \diamond F_{-u_{2}}$ we obtain $f \in F_{u_{1}} \oplus_{c} F_{-u_{2}}$. Therefore,

$$
F_{u}=F_{u_{1}} \oplus_{c} F_{-u_{2}} \text {. }
$$

Let $f \in F_{u_{1}+u_{2}}$. Then $\left\|P_{2}\left(u_{1}-u_{2}\right) f\right\| \leq\|f\|$ and, in view of [2, Lemma 4.2] we obtain

$$
\begin{array}{r}
\left\langle P_{2}\left(u_{1}-u_{2}\right) f, u_{1}+u_{2}\right\rangle=\left\langle f, P_{2}\left(u_{1}-u_{2}\right)^{*}\left(u_{1}+u_{2}\right)\right\rangle= \\
=\left\langle f, P_{2}\left(u_{1}-u_{2}\right)^{*} u_{1}\right\rangle+\left\langle f, P_{2}\left(u_{1}-u_{2}\right)^{*} u_{2}\right\rangle=\left\langle f, u_{1}+u_{2}\right\rangle=1
\end{array}
$$

Therefore, $\left\|P_{2}\left(u_{1}-u_{2}\right) f\right\|=\|f\|$ and from the neutrality of $P_{2}\left(u_{1}-u_{2}\right) f=f$. Thus, from the equalities (6) and (7) we obtain

$$
Z=\overline{\mathrm{sp}} F_{e}=\overline{\mathrm{sp}} F_{u}=\overline{\mathrm{sp}}\left(F_{u_{1}} \oplus_{c} F_{-u_{2}}\right)=\overline{\mathrm{sp}} F_{u_{1}} \oplus \overline{\mathrm{sp}} F_{-u_{2}}=\overline{\mathrm{sp}} F_{u_{1}} \oplus \overline{\mathrm{sp}} F_{u_{2}}
$$


Therefore, using equalities (6) and (7) we obtain that

$$
\begin{gathered}
\operatorname{co}\left(F_{u} \cup F_{-u}\right)=\operatorname{co}\left(\left(F_{u_{1}} \oplus_{c} F_{-u_{2}}\right) \cup\left(F_{-u_{1}} \oplus_{c} F_{u_{2}}\right)\right)= \\
=\operatorname{co}\left(\left(F_{u_{1}} \oplus_{c} F_{u_{2}}\right) \cup\left(F_{-u_{1}} \oplus_{c} F_{-u_{2}}\right)\right)=\operatorname{co}\left(F_{e} \cup F_{-e}\right)=Z_{1} .
\end{gathered}
$$

This means that $u$ is unitary. The proof is complete.

Now we can formulate [12, Theorem 3.1] as follows.

Theorem 4.2. Let $Z$ be a real neutral strongly facially symmetric space with unitary geometric tripotents. Then there exists a measure space $(\Omega, \Sigma, \mu)$ with measure $\mu$ having the direct sum property such that the space $Z$ is isometrically isomorphic to the space $L_{1}(\Omega, \Sigma, \mu)$.

\section{REFERENCES}

[1] Friedman, Y., Russo, B.: A geometric spetral theorem. Quart. J. Math. Oxford. (1986) doi: 10.1093/QMATH/37.3.263

[2] Friedman, Y., Russo, B.: Affine structure of facially symmetric spaces. Math. Proc. Camb. Philos. Soc. (1989) doi: 10.1017/S030500410006802X

[3] Friedman, Y., Russo, B.: Some affine geometric aspects of operator algebras. Pac. J. Math. (1989) doi: $10.2140 /$ pjm.1989.137.123

[4] Friedman, Y., Russo, B.: Geometry of the dual ball of the spin factor. Proc. Lon. Math. Soc. (1992) doi: $10.1112 / \mathrm{plms} / \mathrm{s} 3-65.1 .142$

[5] Friedman, Y., Russo, B.: Classification of atomic facially symmetric spaces. Canad. J. Math. (1993) doi: 10.4153/CJM-1993-004-0

[6] Ibragimov, M., Kudaybergenov, K., Tleumuratov, S., Seypullaev, J.: Geometric Description of the Preduals of Atomic Commutative von Neumann Algebras. Mathematical Notes. (2013) doi: 10.1134/S0001434613050076

[7] Ibragimov, M., Seypullaev, J.: Geometric properties of the unit ball of an SFS-space of finite rank. Uzb. Math. journal. 2, 10-19 (2005)

[8] Ibragimov, M., Seypullaev, J.: Description of the unit balls of the facially symmetric spaces of small dimension. Bulletin of KarSU. 2, 3-5 (2009)

[9] Ibragimov, M., Seypullaev, J.: Description of n-dimensional real strongly facially symmetric spaces of rank n-1. Uzb. Math. journal. 4, 39-46 (2015)

[10] Neal, M., Russo, B.: State space of JB*-triples. Math. Ann. (2004) doi: 10.1007/s00208-003-0495-9

[11] Seypullaev, J.: Geometric characterization of Hilbert spaces. Uzb. Math. journal. 2, 107-112 (2008)

[12] Yadgorov, N., Ibragimov, M., Kudaybergenov, K.: Geometric characterization of $L_{1}$-spaces. Studia Math. (2013) doi: 10.4064/sm219-2-1

Ch. Abdirov 1, Department of Mathematics, Karakalpak State University, Nukus 230113, UZBEKISTAN

E-mail address: karim2006@mail.ru

V.I.Romanovskiy Institute of Mathematics, Uzbekistan Academy of Sciences, 81, Mirzo Ulughbek Street, 100170 TASHKent, UzBekistan

E-mail address: jumabek81@mail.ru 